\title{
BEAM LOSS RESPONSE MEASUREMENTS WITH A LHC PROTOTYPE COLLIMATOR IN THE SPS
}

\author{
Th. Weiler*, G. Arduini, R. Assmann, C. Bracco, H. Braun, B. Dehning, P. Gander, E. B. Holzer, \\ M. Jonker, R. Losito, A. Masi, L. Ponce, S. Redaelli, G. Robert-Demolaize, M. Sobczak, J. Wenninger, \\ CERN, Geneva, Switzerland
}

\begin{abstract}
Beam tests with a Large Hadron Collider (LHC) prototype collimator were performed at the Super Proton Synchrotron (SPS) in autumn 2006. Applying a new collimator control system many new beam measurements were performed. This contribution presents results on collimatorinduced beam loss measurements and their applications to beam-based alignment of collimators and measurements of the beam size and position. Interesting features of the recorded beam loss signals are illustrated and possible impacts for LHC operation are discussed.
\end{abstract}

\section{INTRODUCTION}

In the LHC we store two proton beams with a centre of mass energy of $14 \mathrm{TeV}$. At design luminosity each beam contains $3 \times 10^{14} \mathrm{p}$ which correspond to a stored energy per beam of $360 \mathrm{MJ}$. To handle such beams and protect the super-conducting magnet from unavoidable beam losses a robust and highly reliable collimation system is required. In autumn 2006 a LHC prototype collimator together with the collimation control system were tested in the SPS. Nearly all the functionalities needed for the operation in the LHC was available. It was possible to test commissioning scenarios as needed for the hardware commissioning as well as for the commissioning with beam. Besides this, accelerator physics related measurement were performed, including impedance measurements and nonlinear collimation.

\section{SETUP}

A LHC prototype collimator of the final design was installed in the SPS long straight section 5 (LSS5). The stepping motors, position and temperature sensors were the same as used for the 2004 test beam [1]. Figure 1 shows the installed collimator in LSS5. For measuring the proton losses close to the collimator four LHC beam loss monitors (BLM) were installed downstream of the collimator.

One major goal of the SPS test beam was to test the collimators with the LHC control system. The control path was adapted to SPS needs and environment, but all crucial parts of the LHC control path were implemented [2]. On the low-level side the final motor driver and sensor readout electronics were present. The collimator setting and tuning was done with a dedicated graphical user interface as it will be used in the LHC control room, see figure 2. Through this

\footnotetext{
*Thomas.Weiler@cern.ch
} 01 Circular Colliders

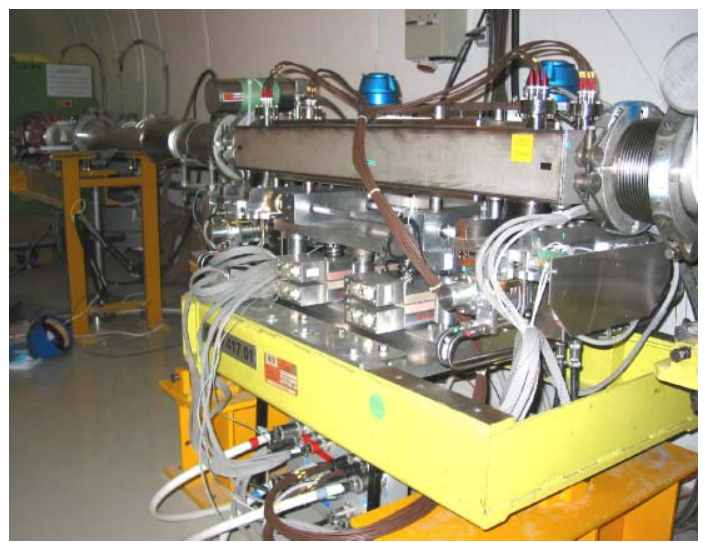

Figure 1: LHC collimator prototype installed in the LSS5 of SPS.

interface it was possible to view the output of the sensors and beam loss monitors online. This provided a powerful tool to position the jaws and analyse their impacts on the beam.

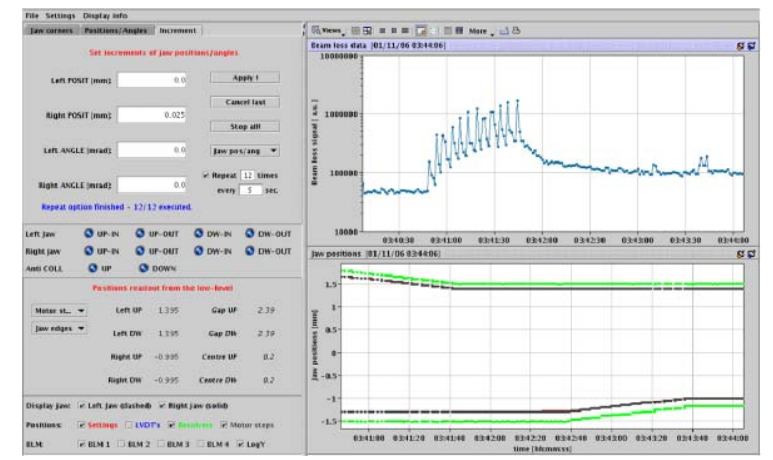

Figure 2: Main view of the graphical user interface used for steering the LHC collimator in the control room.

The beam energy used through the tests was $270 \mathrm{GeV}$, only the intensity was varied according to the needs of the studies. For the nominal low intensity runs one bunch containing $1.15 \times 10^{11} \mathrm{p}$ was used and at high intensity 288 bunches containing $1.15 \times 10^{11} \mathrm{p}$ per bunch were used.

\section{RESULTS}

Prior to the tests with beam the collimator control software was tested and the jaws were calibrated using the precisely measured positions of the end switches as reference points. By moving the collimator jaws to the calibrated end

T19 Collimation and Targetry 
switches and anti-collision switches the correct readout of these switches and the reliability of the controls software interlock system was verified. The readout of the position sensor, which were not of the final LHC type, showed a high noise level of $\approx 100 \mu \mathrm{m}$. Since this is not sufficient for LHC operation and the planned accelerator physics test (e.g. impedance measurements) the position the motor readings were used as reference throughout the tests. A later test conducted in the extraction line TT40 with with the final position sensors showed that a $5 \mu \mathrm{m}$ precision can be achieved by using the final sensors.

After the controls software and the collimator were commissioned, the measurements with beam started by the centring of the two jaws around the beam. One beam based alignment alignment method is described below [4]. One jaw is moved to the beam until it starts cleaning the halo. This jaw is moved in small steps, typically 10 to $20 \mu \mathrm{m}$, until consecutive loss signals seen by the beam loss monitors have the same size. Then the other jaw is moved to the beam until it touches the beam, the geometrical middle of the two jaw positions defines the beam centre. With alternating movements of the two jaws and reduced step size the beam centre position measurement can be refined. Figure 3 shows the jaw positions and signals of the beam loss monitors for the beam based alignment done during the beam test. The average of the measured beam centre position was $0.199 \pm 0.01 \mathrm{~mm}$. Attempts to calibrate the collimator angle with beam were inconsistent and provided not conclusive results. Further studies are ongoing.

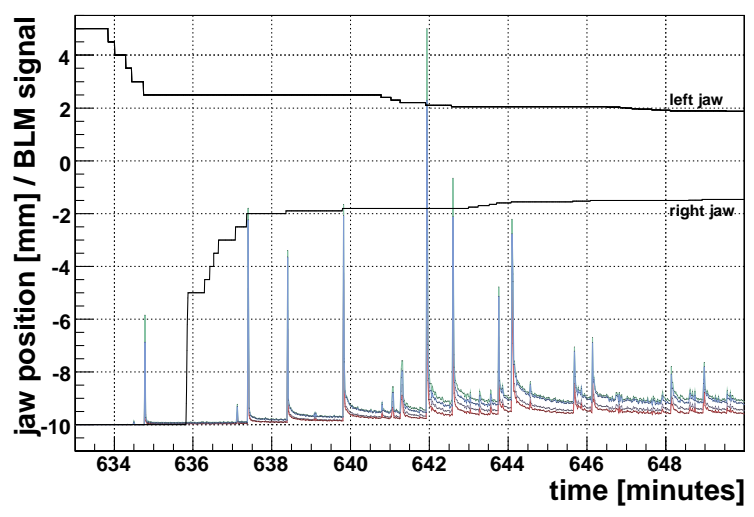

Figure 3: Beam based alignment. One collimator jaw is moved towards the beam in small steps until loss peaks have comparable size. Then the second jaw is moved in small steps until it touches the beam. The measurement is refined using smaller steps and comparing the loss signals of alternating jaw movements.

Another possibility for measuring the beam centre is to fully scrape away the beam and plot the beam current versus the jaw position [3]. The beam centre corresponds to the jaw positron where the beam current vanishes. Figure 4 shows the the decreasing of the beam current according to the scraping of the beam with one jaw. This method re01 Circular Colliders quires a precise timing information on the sensor readings. The timestamping of the readings was not yet implemented at the low-level but only in the middle-level controls. This can result in time-shifts of up to $0.5 \mathrm{~s}$ and a large error in determining the beam centre results using this method. In addition the scraping of the beam is also suited to measure the beam size by applying a Gaussian fit to this curve. The obtained value of $(854 \pm 26) \mu \mathrm{m}$ is in quite good agreement with the wire scanner measurements.

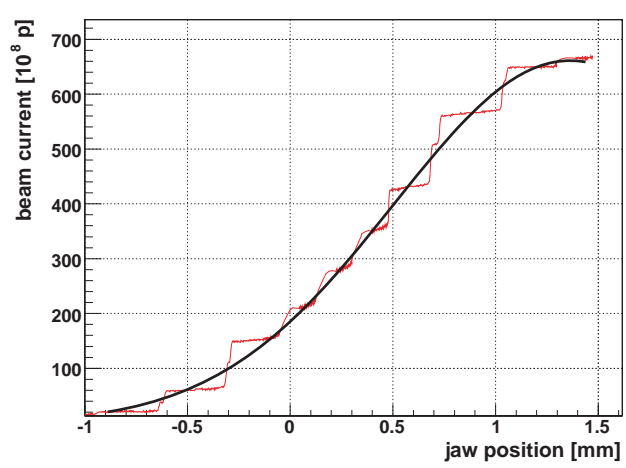

Figure 4: This graph shows the decrease of the beam current according to the scraping of the beam by one jaw. Movement of the jaw is done in small steps. By fitting a Gaussian to this curve the beam size can be evaluated to $\approx(854 \pm 26) \mu \mathrm{m}$.

Figure 5 shows the response of the beam loss monitor signal to a jaw moving into the beam, staying there for approximately $30 \mathrm{~s}$ and being retracted afterwards. The signal of the beam loss monitor immediately raises when the jaw touches the beam, but the decay of the signal lasts approximately 20 seconds. This behaviour was also seen in the 2004 collimator beam test [5]. The long decay time of the signal does not originate from the readout electronics and beam loss monitor. This was extensively studied after the 2004 test, in addition the beam loss monitors used for this two beam-tests are different. To exclude that this effect is related to RF-noise, the RF-cavities were switched off and tail measurements were repeated. The behaviour of the signal tails did not change, leading to the conclusion that this is a real beam dynamics effect related to coupling of the two planes.

In addition to the losses seen by jaw movements, also the losses related to the change in the machine tune were studied. Figure 6 shows the losses correlated to the tune change. After each tune change the jaw was moved towards the beam to study the behaviour of the loss tail. Figure 7 shows a loss peak caused by the tune change followed by to peaks not related to a jaw movement or tune change. This was observed for a tune of $Q_{x}=0.20$ and $Q_{x}=0.24$, the origin of this effect is still under investigation.

Beside the measurements with the LHC-type beam loss monitors, loss maps using the SPS beam loss monitors located around the ring have been recorded. Figure 8 shows 


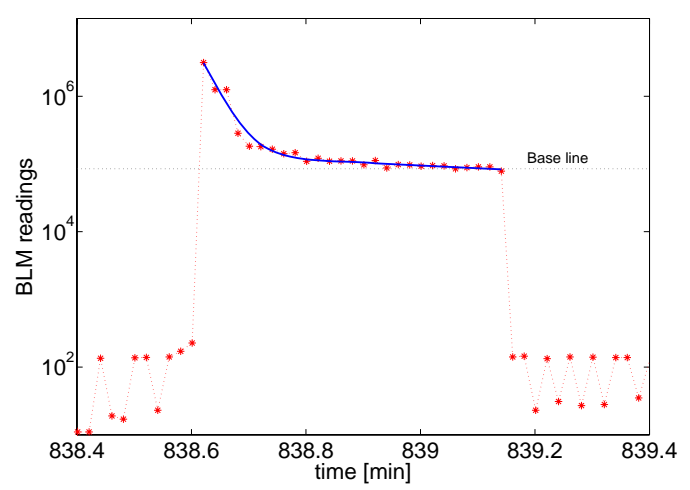

Figure 5: Typical response of beam loss monitor signal to jaw movement. After the loss signal reached its maximum at 838.63 minutes a slow decay of the signal is observed.

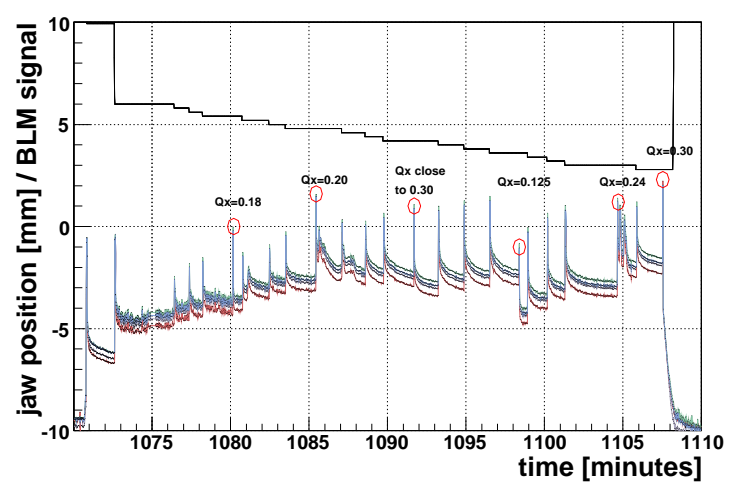

Figure 6: The plot shows the position of one jaw (the other was far out of the beam) and corresponding loss signals in the beam loss monitors when the tune of the accelerator is changed. At every tune change a loss peak was observed without moving the jaw.

the integrated losses around the ring for a time interval of $25 \mathrm{~s}$. The high loss peak corresponds to losses generated by the collimator installed in LSS5, but also in other location an accumulation of losses can be detected.

\section{CONCLUSIONS}

During the 2006 SPS test-beam the crucial parts of the collimator control system were extensively tested. The main functionality needed for the hardware commissioning and later on the commissioning with beam has shown to be operational. The possibility to measure the beam centre with an accuracy of $10 \mu \mathrm{m}$. by beam based alignment has been experimentally demonstrated. Also the scraping of the beam provides useful tool for beam position and size measurements. However it showed that a proper timing information on the jaw position is essential. In addition slow and fast beam loss monitor signal were recorded around the ring and several measurements to determine the collimators impedance were performed [6].

01 Circular Colliders

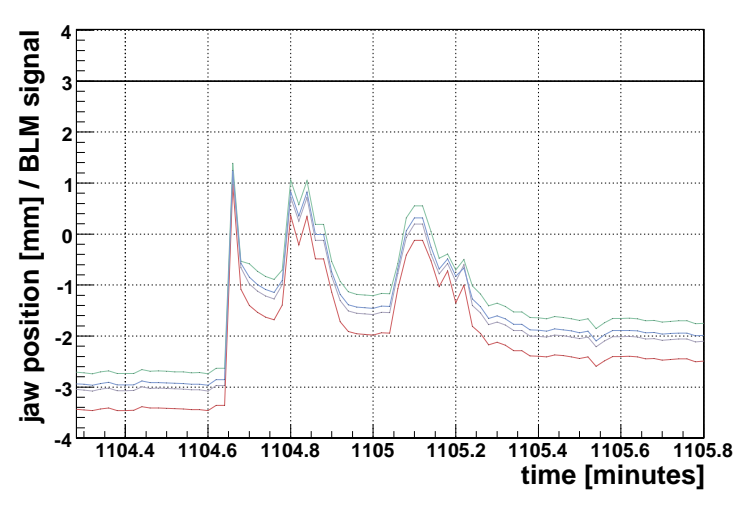

Figure 7: The plot shows a loss peak corresponding to the tune change, followed by two peaks. The time between the peaks is in the order of $10 \mathrm{~s}$.

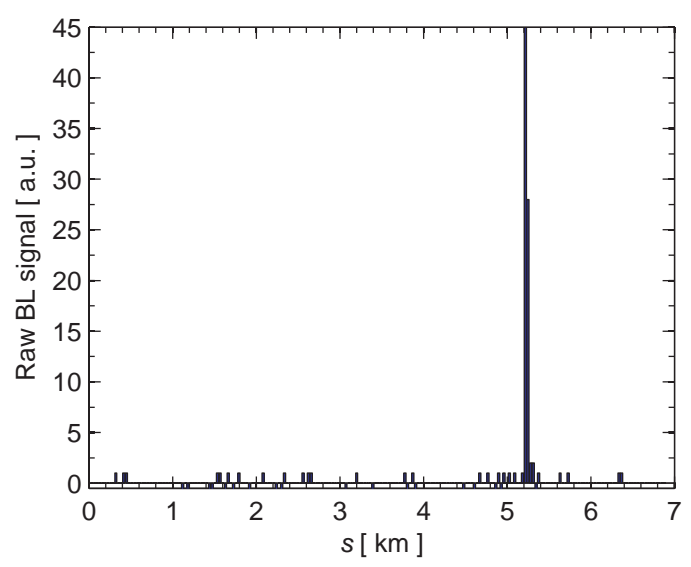

Figure 8: This plot shows the integrated losses around the SPS for a time interval of $25 \mathrm{~s}$. The high loss peak corresponds to losses generated by the collimator installed in LSS5.

\section{REFERENCES}

[1] R. Assmann et al., "LHC Collimation: Design and Results from Prototyping and Beam Tests", CERN-LHC-PROJECTREPORT-850, 2005.

[2] S. Redaelli et al., "The LHC Collimator Controls Architecture", PAC07, Albuquerque, New Mexico, 2007.

[3] A. Jansson et al., "Collimator scans to measure the Tevatron emittance", Tevatron beam study report, 2003.

[4] S. Redaelli, et al., "LHC Aperture and Commissioning of the Collimation System",Chamonix XIV, 2005.

[5] G. Robert-Demolaize, "Design and Performance of the LHC Collimation System", CERN-Thesis-2006-069, 2006.

[6] E. Metral et al., "Transverse Impedance of LHC Collimators", PAC07, Albuquerque, New Mexico, 2007. 